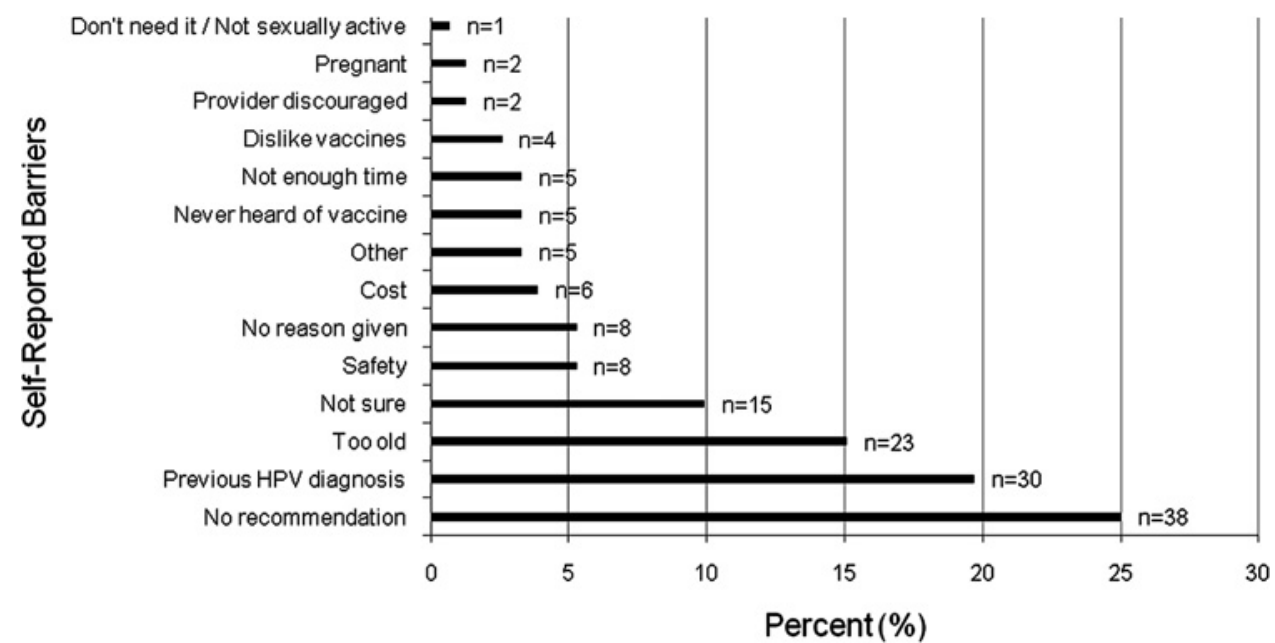

Abstract P1-S6.43 Figure 1 Self-reported barriers to hpv vaccination among women with precancerous cervical lesions ( $n=152$ ).

Methods Telephone interviews were conducted among women diagnosed with precancerous cervical lesions and reported to the CT Department of Public Health for routine surveillance during 2008-2010. The sample consisted of 269 women ages 18-27 years (age-eligible for catch-up vaccination after licensure) including $77 \%$ white, $15 \%$ black, and $17 \%$ Hispanic; median age was 23 years.

Results Overall, $43 \%$ of women reported having received $\geq 1$ dose of HPV vaccine (mean age at vaccination 22 years). Publicly insured and uninsured women were significantly less likely than privately insured women to have received vaccine $(23 \%$ and $15 \%$ vs $52 \%$, $\mathrm{p}<0.05$ for both), and black women were marginally less likely to receive vaccine compared to white women ( $31 \%$ vs $48 \%, \mathrm{p}=0.06$ ). There was no significant difference for ethnicity. The most common self-reported barrier to vaccination was lack of provider recommendation (25\%). Other common self-reported reasons were previous HPV diagnosis (20\%) and being too old (15\%). Women who did not discuss HPV vaccine with a provider were more likely to have not received vaccine compared to women who discussed vaccine (95\% vs 44\%, p<0.001) see Abstract P1-S6.43 Figure 1.

Conclusions Provider interventions may be necessary to assure catch-up vaccine is offered to eligible women. A common reported barrier was being too old, yet all women in this sample were ageeligible for vaccination. Previous HPV infection was another common barrier, yet this is not a contraindication for vaccination to protect against infection from other HPV types. In particular, greater efforts are required to administer catch-up vaccine to low-income and black women. Providing vaccine for underinsured women in this age group will be a challenge because they are not eligible for some state or federal vaccination programs (eg, Vaccines For Children).

\section{P1-S6.44 HIV VACCINE CLINICAL TRIAL ADHERENCE AND RETENTION: HIGH-RISK DRUG-USING WOMEN}

doi:10.1136/sextrans-2011-050108.268

J Becher, S Chhatre, M Eisenberg, D Fiore, T Dominique, D Dunbar, I Frank, D Metzger. University of Pennsy/vania, Philadelphia, USA

Background Clinical trial protocol adherence and retention are often considered challenges that are especially difficult to achieve among certain high-risk populations. The HIV infection rate among heterosexual African-American women is increasing, making their participation in clinical trials of HIV behavioural and biomedical prevention interventions more important. We identify drug use and sex risk factors associated with adherence to protocol and study retention among this population during the course of an HIV vaccine trial.
Methods Data from participants at the Philadelphia, PA site for the HIV Vaccine Trials Network (HVTN) 502 study testing the Merck Adenovirus 5 gag-pol-nef HIV vaccine were used to examine factors associated with adherence (receipt of three vaccines) and retention (present for 12-month appointment). Enrollees included $123 \mathrm{HIV}$ negative women $18-45$ years of age reporting regular use of crack cocaine and frequent unprotected sex, who were randomly assigned to receive three injections of either the study vaccine or a placebo vaccine.

Results Study participants had a mean age of 37 years and $91 \%$ were African-American. Overall adherence to study protocol was $89 \%$ and study retention at one year was $93.5 \%$. Analyses found no association between drug use and high-risk sex behaviours and poorer rates of adherence and retention. In fact, participants who used recreational drugs at baseline were more likely than those who did not to adhere to study protocol $(92 \%$ vs $75 \%$, p < 0.10$)$. Participants who reported use of injectable contraceptives at baseline were less likely than those who did not to be retained in the study at 1 year $(57 \%$ vs $96 \%, p<0.01)$. Other measures of drug use (whether injected recreational drugs, and used crack cocaine, speed and other drugs) and sex risk (whether had a STI, exchanged vaginal, oral or anal sex for money, drugs or services, used different methods of birth control, the number of male sex partners, whether aware of partner's HIV status and whether had protective and unprotected vaginal, anal or oral sex with them) were not associated with adherence and retention.

Conclusions Factors commonly assumed to interfere with trial participation were not associated with adherence to study protocol or retention. These findings suggest that drug use and sexual risk behaviours do not impede completion of vaccinations and protocol required visits in clinical trials of experimental HIV vaccines.

\section{Epidemiology poster session 6: Preventive intervention: Community action \\ P1-S6.45 IMPACT OF A COMMUNITY LEVEL, DIFFUSION BASED HIV/ STI INTERVENTION ON HETEROSEXUALLY-IDENTIFIED, SOCIALLY MARGINALISED MEN IN URBAN, COASTAL PERU}

doi:10.1136/sextrans-2011-050108.269

${ }^{1} \mathrm{~K}$ Konda, ${ }^{2} \mathrm{~A}$ Lescano, ${ }^{3} \mathrm{D}$ Celentano, ${ }^{4} \mathrm{~A}$ Maiorana, ${ }^{5} \mathrm{X}$ Salazar, ${ }^{5} \mathrm{~A} \mathrm{M}$ Rosasco, ${ }^{2} \mathrm{~S}$ Montano, ${ }^{2} \mathrm{~T}$ Kochel, ${ }^{2} \mathrm{E}$ Hall, ${ }^{1} \mathrm{~T}$ Coates, ${ }^{5} \mathrm{C}$ Caceres, ${ }^{4} \mathrm{~S}$ Kegeles, ${ }^{6} \mathrm{NIMH}$ 
Collaborative HIV/STD Prevention Trial Group. ${ }^{1}$ UCLA, Lima, Peru: ${ }^{2}$ NAMRU-6, Callao, Peru; ${ }^{3} J o h n s$ Hopkins University, Baltimore, USA; ${ }^{4}$ UCSF, USA; ${ }^{5}$ UPCH, Lima, Peru; ${ }^{6} \mathrm{NIMH}$, USA

Objectives The NIMH Collaborative HIV/STD Intervention Trial implemented a 2-year intervention based on the theory of diffusion of innovations to decrease unprotected sex with non-primary partners among high-risk populations including heterosexually-identified, socially marginalised men in urban, coastal Peru.

Methods 20 communities in 3 cities were randomised to the intervention or comparison arm. In intervention communities, $15 \%$ of the target population was trained as Community Popular Opinion Leaders (CPOLs) to deliver HIV prevention messages to their peers. In all communities, yearly assessments of the study cohort, independent of the intervention, gathered information on sexual behaviour, provided pre-test counselling, and testing for HIV/STIs at baseline, 1 year, and 2 years after randomisation. This analysis included the 2146 heterosexually-identified men who fulfilled the trial's inclusion criteria (frequented community social venues, were aged 18 to 40, and had sex in the past 6 months).

Results Unprotected sex with a non-primary partner decreased by $10 \%$ in both study arms among heterosexually identified men. Almost half of the participants, in both the intervention and in the comparison arms, reported unprotected intercourse with a nonprimary partner at the final follow-up (47.6\% vs $48.7 \%, \mathrm{p}=0.694$ ) and there was no difference after controlling for baseline behaviour, OR 1.04, (95\% CI 0.79-to 1.38). STI incidence was also did not differ by randomisation condition, IRR 1.14 (95\% CI 0.77 - to 1.68) comparing incidence in intervention vs control communities.

Discussion We speculate that the lack of effect of the intervention among the heterosexually-identified men may be attributable to 1) an insufficiently strong intervention approach, 2) this group of men did not feel vulnerable to HIV or STI, or 3) the repeated biomedical testing and ancillary required counselling were as potent as the CPOL intervention. Future HIV prevention trials with this population should try to tailor the intervention to this group's risk perceptions and behaviours.

\section{P1-S6.46 THE LONG-TERM IMPACT OF THE MEMA KWA VIJANA ADOLESCENT SEXUAL AND REPRODUCTIVE HEALTH INTERVENTION: EFFECTS OF DOSE AND TIME SINCE EXPOSURE TO INTERVENTION}

doi:10.1136/sextrans-2011-050108.270

${ }^{1} \mathrm{~A}$ M Doyle, ${ }^{1} \mathrm{H}$ A Weiss, ${ }^{2} \mathrm{~K}$ Maganja, ${ }^{3} \mathrm{~S}$ McCormack, ${ }^{1} \mathrm{D}$ Watson-Jones, ${ }^{2} \mathrm{~J}$ Changalucha, ${ }^{1} \mathrm{R}$ J Hayes, ${ }^{1} \mathrm{D}$ A Ross. 'London School of Hygiene \& Tropical Medicine, London, UK; ${ }^{2}$ National Institute for Medical Research, Mwanza Centre, United Republic of Tanzania; ${ }^{3}$ MRC Clinical Trials Unit, UK

Background Despite recent decreases in HIV incidence in many countries in sub-Saharan Africa, there is little evidence that specific behavioural interventions have led to a reduction in HIV among young people. Further and wider-scale decreases in HIV will require better understanding of when behaviour change occurs and why. The MEMA kwa Vijana adolescent sexual and reproductive health intervention has been implemented in rural Mwanza, Tanzania since 1999. A long-term ( $9 \mathrm{yr}$ ) evaluation found that the intervention had improved knowledge, reported attitudes to sex and some sexual risk behaviours but not HIV or HSV2 prevalence. An evaluation of impact in 2001 found strong evidence that receiving more years of the intervention (dose) was associated with a larger impact on some outcomes. We explored whether there was longterm differential intervention impact according to age, marital status, dose of intervention, or time since last exposure to the inschool component of the intervention.
Methods In 2007, a cross-sectional survey was conducted in the 20 MEMA kwa Vijana trial communities among 13814 young people (15-30yrs) who had attended years 5-7 at intervention or comparison schools between 1999 and 2002. Only outcomes for which the intervention was shown to have an impact in 2001 or 2007 were included in this subgroup analysis that is, knowledge, reported attitudes, selected reported behaviours (age at first sex; number of partners; condom use). Data were analysed using clusterlevel methods for stratified cluster-randomised trials with interaction tests to determine if intervention impact differed according to subgroup.

Results Taking into account multiplicity of testing, concurrence with a priori hypotheses and consistency within the results no strong effect modifiers emerged. There was some evidence of a doseresponse effect. Specifically, impact on pregnancy knowledge increased with increasing high-quality dose of the intervention in both sexes, especially among males (Abstract P1-S6.46 table 1). There was some evidence of an impact of the high-quality dose of intervention on overall sexual attitudes among females. The consistency of these findings suggests that they are highly plausible.

Abstract P1-S6.46 Table 1 Impact of intervention in 2007/8 according to number of years of exposure to in-school intervention (1999-2002)

\begin{tabular}{|c|c|c|c|c|c|}
\hline \multirow[b]{2}{*}{ Outcome } & \multirow[b]{2}{*}{ Overall } & \multicolumn{3}{|c|}{ Years of in-school intervention ('99-'02) } & \multirow{2}{*}{$\begin{array}{l}\text { Test for } \\
\text { trend } \\
\text { (p value) }\end{array}$} \\
\hline & & $1 \mathrm{yr}$ & 2 yrs & $3+y r s$ & \\
\hline \multicolumn{6}{|c|}{ HIV acquisition knowledge (\% with all 3 responses "correct") } \\
\hline Male & $1.11(0.99,1.23)^{*}$ & 1.09 & 1.12 & 1.12 & 0.56 \\
\hline Female & $1.11(1.00,1.24)^{*}$ & 1.12 & 1.11 & 1.12 & 0.85 \\
\hline \multicolumn{6}{|c|}{ STD acquisition knowledge (\% with all 3 responses "correct") } \\
\hline Male & $1.18(1.04,1.34)^{* *}$ & 1.19 & 1.20 & 1.16 & 0.70 \\
\hline Female & $1.24(0.97,1.58)^{*}$ & 1.22 & 1.21 & 1.32 & 0.32 \\
\hline \multicolumn{6}{|c|}{ Pregnancy prevention knowledge (\% with all 3 responses "correct") } \\
\hline Male & $1.19(1.12,1.26)^{* *}$ & 1.13 & 1.19 & 1.25 & $0.0001^{* *}$ \\
\hline Female & $1.17(1.06,1.30)^{* *}$ & 1.12 & 1.20 & 1.19 & $0.03^{* *}$ \\
\hline \multicolumn{6}{|c|}{ Attitudes to sex (\% with all 3 responses "correct") } \\
\hline Male & $1.31(0.97,1.77)^{*}$ & 1.26 & 1.33 & 1.38 & 0.15 \\
\hline Female & $1.09(0.67,1.77)$ & 0.78 & 1.23 & 1.25 & $0.008^{* *}$ \\
\hline
\end{tabular}

Conclusions The desirable long-term impact of the MEMA kwa Vijana intervention did not vary greatly according to the subgroups examined. From a programme implementation point of view this suggests that the intervention can have an impact on a broad crosssection of young people in rural Mwanza.

\section{P1-S6.47 DESCRIPTION AND EVALUATION OF A REGIONAL CONDOM ACCESS PROGRAM FOR COMMUNITY ORGANIZATIONS}

doi:10.1136/sextrans-2011-050108.271

${ }^{1} \mathrm{G}$ Petit, ${ }^{2} \mathrm{~A}$ Bigras, ${ }^{2} \mathrm{C}$ Dubé, ${ }^{2} \mathrm{~F} \mathrm{C}$ Malo, ${ }^{2} \mathrm{M} \mathrm{C}$ Surprenant, ${ }^{2} \mathrm{M}$ Salvail, ${ }^{1} \mathrm{M}$ Gagnon. ${ }^{1}$ Direction de santé publique et de l'évaluation, Canada; ${ }^{2}$ Université de Sherbrooke, Sherbrooke, Canada

Background As observed in the rest of Quebec province, the number of STD declared to public health authorities in Estrie have been increasing in the last years. It has also been evaluated that condom use in this region is not as high as expected, particularly by young people. The Estrie region began a program in 2008 to favour free access to condoms for community organizations in contact with groups known as vulnerable to STDs. Purpose Describe and evaluate the implementation of the program after a period of 3 years. The regional program aim is to provide free condoms to 